\title{
A Distributed Proxy System for Provisioning Immersive Audio Communication to Massively Multi-player Games
}

\author{
Cong Duc Nguyen, Farzad Safaei, Paul Boustead \\ Telecommunications and Information Technology Research Institute \\ University of Wollongong, Australia \\ cong,farzad,paul@titr.uow.edu.au
}

\begin{abstract}
This paper evaluates the suitability of a distributed proxy architecture for the provision of an immersive audio communication service for massively multi-player online games. The immersive audio communication service enables each avatar to hear a realistic audio mix of conversations in its hearing range. The proxy architecture is presented to address some limitations of previously proposed architectures, namely peerto-peer and central server. We have developed a simulation model to evaluate the performance these delivery architectures in different game delivery scenarios. In particular, the bandwidth efficiency of network multicast for the proxy architecture in different avatar grouping behaviours and player distribution scenarios is studied. In addition, we investigate the impact of varying the number of proxy servers on the game communication delays.
\end{abstract}

Categories and Subject Descriptors: C.2.4 [Distributed Systems]: Distributed applications

General Terms: Performance, Design, Economics

Keywords: Massively multi-player games, immersive audio, provisioning, multicast, simulation

\section{EXTENDED ABSTRACT}

Natural voice communication in networked games is likely to enhance player satisfaction and improve market penetration of many games. However, in massively multiplayer games, inter-person audio communication is still clumsy and unnatural, primarily text-based and augmented (in some cases) by limited voice communications such as a party line.

In earlier work [1], we introduced the concept of immersive audio communication service for these games. This service allows each the avatar to hear a personalised mix of voices of other avatars in its hearing range, spatially placed and attenuated according to distance. A peer-to-peer and a central server architectures have been proposed for this service. However, the peer-to-peer architecture faces serious access bandwidth scalability problems while the central server architecture is subject to high delay, processing scalability and single point of failure. In this paper, a distributed proxy architecture is introduced to overcome limitations of these architectures. In this architecture, a group of participants in a given geographical location are assigned to a nearby server. This server, which is referred to as a proxy, is responsible for the creation audio scenes for its attached participants. These proxies can send audio flows either using unicast or network multicast.

Copyright is held by the author/owner.

SIGCOMM'04 Workshops, Aug. 30+Sept. 3, 2004, Portland, Oregon, USA. ACM 1-58113-942-X/04/0008.
We have developed a simulation environment that creates both the physical world (geographic distribution of participants and the Internet topology model) and the virtual world. The virtual world is created based on three main avatar aggregation behaviours: loners, clans and crowds. Loners represent isolated avatars which are relatively far away from each other; a clan represents a medium size group, such as a hunting group; a crowd represents a very large group, such as a market place. A parameter loosely referred to as "correlation" is introduced to specify the tendency that players from close geographic regions to aggregate together in the virtual world due to language, culture and lifestyle preferences.

Our simulation results indicate that if a larger number of proxy servers are available over the Internet, the distributed proxy architecture would be a cost effective delivery architecture since it reduces the delay of the central server architecture and solves access bandwidth problems of the peerto-peer architecture. However, if this is not the case, other architectures should be considered. Therefore, in loner based games, the peer-to-peer architecture would be favored due to low delays in direct paths between avatars and acceptable levels of access bandwidth, however, security/anonymity issues must be addressed. For games consisting of crowds and clans, the distributed proxy architecture is most appropriate due to improvements in communication delays over the central server, especially, when there is a correlation between the virtual world and the physical world. However, if the number of available proxy servers is small, the central server may be preferred. In many games, where all different player characteristics may be apparent in different parts of a virtual world, a hybrid architecture would be most suitable, in which, parts of the virtual world that mainly consist of loners would use the peer-to-peer architecture, while crowds or clans would either use the distributed proxies or the central server.

Another recommendation is on the advantages of network multicast over unicast in different game scenarios. Multicast is effective only when the average number of avatars in hearing range is large (i.e crowds). In these scenarios, when game players are connected to a small number of Internet service provider point of presences, the bandwidth cost saving of multicast is significant. However, when game players are widely spread, the efficiency of multicast reduces significantly.

\section{REFERENCES}

1] C. Nguyen, F. Safaei, D. Platt. On the provision of immersive audio communication to massively multi-player online games. In Proc. IEEE ISCC, 2004, pp. 1000-1005.

\section{Acknowledgments}

This work is supported by Co-operative Research Centre for Smart Internet Technology (SITCRC), Australia. 